\title{
A Comparative Study of Otsu Thresholding and K-means Algorithm of Image Segmentation
}

\author{
Arpan Kumar, Anamika Tiwari
}

\begin{abstract}
In computer vision the image segmentation is one of the most important used approach for the partitioning of digital image in the set of pixels on the basis of similarity and discontinuity. The main aim of image segmentation is to make the image analysis easy and meaningful. Among all the image segmentation processes, Otsu algorithm and K-means method are one of the best image thresholding methods among all due to their easier calculation process and methodology. Otsu algorithm is an instinctive threshold picking region based segmentation process. In this paper, Otsu image segmentation algorithm and K-means method with its pros and cons are comparatively reviewed and analyzed.
\end{abstract}

Index Terms - Thresholding, K-means, Image Processing, Otsu Algorithm, Image Segmentation.

\section{INTRODUCTION}

Image segmentation is an advance approach of the bifurcation of the digital image into various different set of pixels, generally called segments. The principal purpose of image segmentation approach in image processing is to make the image representation so easier that it can be analyzed without any hassle. Image segmentation has its various applications like detecting the tumors in organs, artificial intelligence based surgery, object detection in images, fingerprint recognition, face detection, video surveillance, medical imaging etc.

Image segmentation approach is classified on the basis of two characteristics discontinuity and similarity [1]

\section{A. Discontinuity detection based approach}

In this method the image is severed into regions based on the discontinuity. The edge and discontinuity based detection is a segmentation approach in which components derived from discontinuity of intensity, are identified and linked with the outers of regions [2].

\section{B. Similarity detection based approach}

In similarity detection based approach of image segmentation the digital image is segmented in different regions on the basis of similarities like utilizing similar set of pixels. Under this approach various methods like thresholding techniques, clustering techniques, region merging, region splitting and region growing techniques are categorized [2].

Arpan Kumar, M.Tech Scholar, Department of CSE, Bhabha Institute of Technology, Kanpur, India.

Anamika Tiwari, Assistant Professor, Department of CSE, Bhabha Institute of Technology, Kanpur, India.

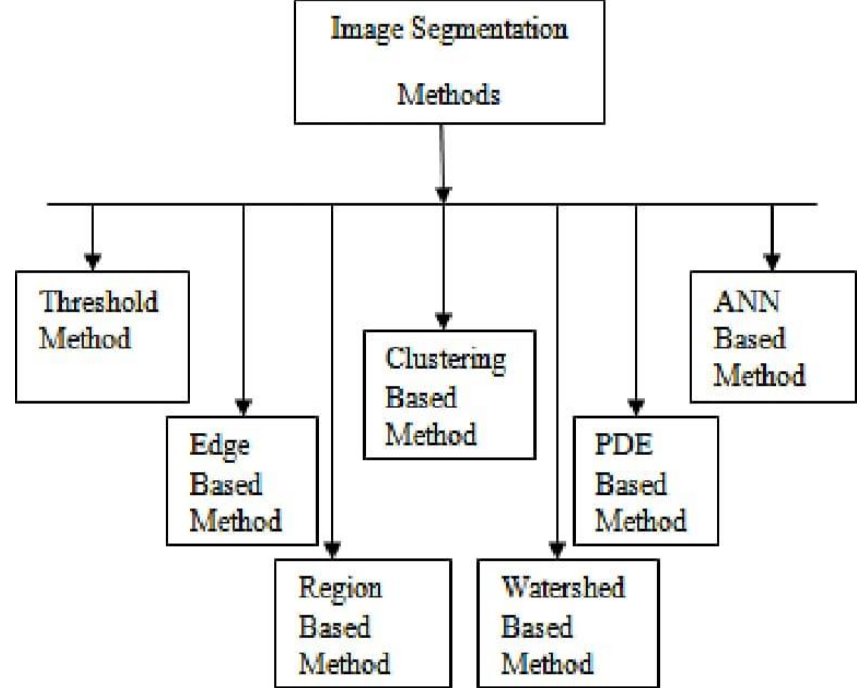

Fig.1- Image Segmentation Techniques

Thresholding is vitally used image segmentation approach to get the image partitioned in various types of segments. The thresholding technique refers the process of opting the optimal threshold value so that the bifurcation if the image on the basis of it can be done and the analysis of digital image on the basis of grey-level distribution can be done. In image thresholding algorithms the histogram of the image plays a prime role as a tool for algorithm development.

Thresholding technique can be defined as:

$$
\mathrm{T}=\mathrm{M}[\mathrm{x}, \mathrm{y}, \mathrm{p}(\mathrm{x}, \mathrm{y}), \mathrm{f}(\mathrm{x}, \mathrm{y})]
$$

Where,

$\mathrm{T}$ denotes the threshold;

$\mathrm{f}(\mathrm{x}, \mathrm{y})$ is the grey value of point $(\mathrm{x}, \mathrm{y})$;

$\mathrm{p}(\mathrm{x}, \mathrm{y})$ is the local property of the point $(\mathrm{x}, \mathrm{y})$;

Thresholding is further divided into two categories as follows:

\section{A. Global thresholding}

Global thresholding refers that it is an approach in which the threshold $\mathrm{T}$ is only derived by focusing the grey level value of the image and it exclusively relates the pixel characteristics.

\section{B. Local thresholding}

Local thresholding states that if the value of threshold $\mathrm{T}$ depends on the grey level value and local properties of the image pixel the that is known as local thresholding technique. In this approach of thresholding the various thresholds are opted from the various respective segmented regions of the image [3]. 


\section{LITERATURE REVIEW}

Zhong Qu and Li Hang [4] proposed 1-Dimensional Otsu algorithm which is being widely used because its simple, efficient and reliable in nature. 1-Dimensional Otsu algorithm merely examines the grey level information of the image pixel disregarding spatial neighbourhood information which can cause low quality segmentation. According to the L. Dongju and Y. Jian the main function of Otsu method is like as the K-means method of multilevel thresholding. Both the approaches are focused on the within class variance minimization. The Otsu method of image segmentation performs the segmentation of digital image using grey level histogram while this is not so in K-means method of image segmentation. Otsu method uses the global thresholding while K-means method uses the local thresholding method of the image segmentation. Since K-means does not need the histogram for calculation so it can be enhanced to multilevel thresholding, two-dimensional thresholding and three-dimensional thresholding. The clustering outcomes of the K-means are obtained in the ordered initial centroid with respect to 1-Dimensional data set [5].

\section{OTSU METHOD}

In image processing Otsu algorithm, proposed by Nobuyuki Otsu performs the automatic clustering based image threholding and performs the conversion of digital grey level image in binary image. In this method it is presumed that it considers that there are two classes of pixels that are foreground pixels and background pixels of the image. The optimum thresholding is calculated by distinguishing the two classes so that the minimum class variance can be obtained. The Otsu algorithm is as given follows:

\section{Algorithm}

1. Draw the histogram of the digital image and calculate the various intensity levels.

2. Initialize $\omega_{\mathrm{i}}(0)$ and $\mu_{\mathrm{i}}(0)$.

3. Calculate all possible threshold values from $\mathrm{t}=$ $1 \ldots . . . n$

Update $\omega_{\mathrm{i}}$ and $\mu_{\mathrm{i}}$ for all possible values Calculate $\sigma_{\mathrm{b}}^{2}$ for all threshold values $\mathrm{t}$.

4. Desired threshold relates to maximum of $\sigma_{b}^{2}$; [6].

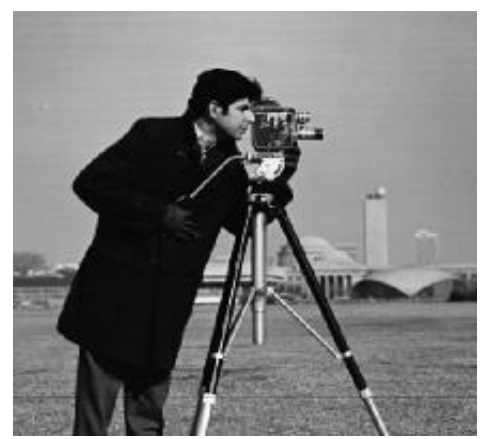

Fig. 2- Original Image

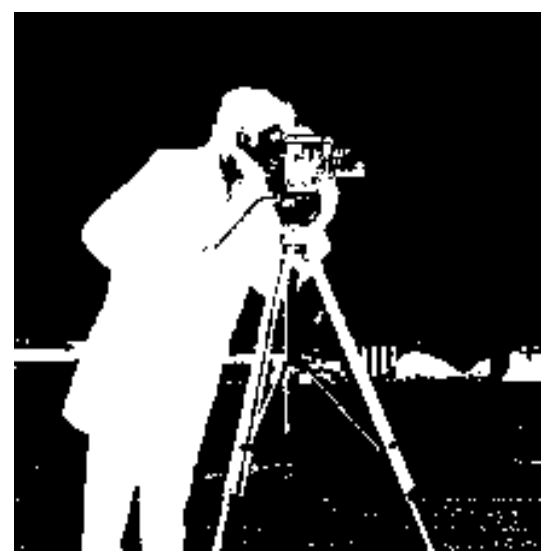

Fig. 3- Otsu algorithm threshold image

\section{K-MEANS METHOD}

$\mathrm{K}$-means algorithm of image segmentation is a kind of supervised algorithm which segments the interest region from the background. In the K-means method of image segmentation firstly the image is contrast stretched so that the good quality image for segmentation can be obtained. In $\mathrm{K}$-mean method collection of data is classified into $\mathrm{k}$ number of data groups. K-means method has two main isolated steps. In first step calculates for $\mathrm{k}$ centroids and in the second step this method considers each point in the cluster which is nearest to the centroid from the respective data point. In $\mathrm{K}$-means method the distance of nearest centroid is generally calculated using Euclidean distance [7].

Let us consider an image (x, y) which is to be used for clustering as to form $\mathrm{k}$ number of clusters. Let $\mathrm{p}(\mathrm{x}, \mathrm{y})$ be a pixel which is to be clustered and $C_{k}$ be the centroid. The algorithm for $\mathrm{K}$-means clustering is as follows:

1. Initialize the desired number of clusters $\mathrm{k}$ and centroid $\mathrm{C}_{\mathrm{k}}$.

2. For every pixel, calculate the Euclidean distance between each pixel and centroid.

3. On the basis of distance $d$, allocate the all pixels to nearest centroid.

4. After assigning all the pixels, recalculate the centroid $\mathrm{C}_{\mathrm{k}}$.

5. Repeat the step 1 to 4 until the error value is satisfied.

6. Form the reshaped image using cluster pixels.

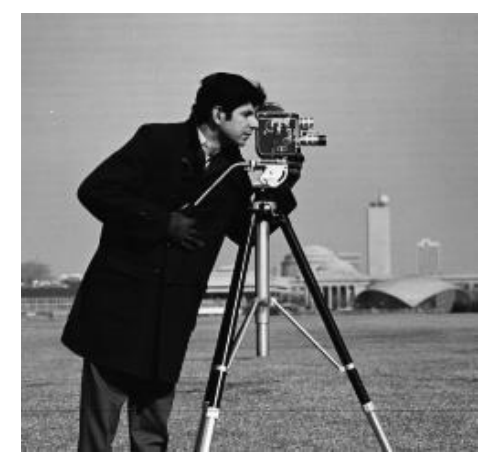

Fig. 4- Original Image 


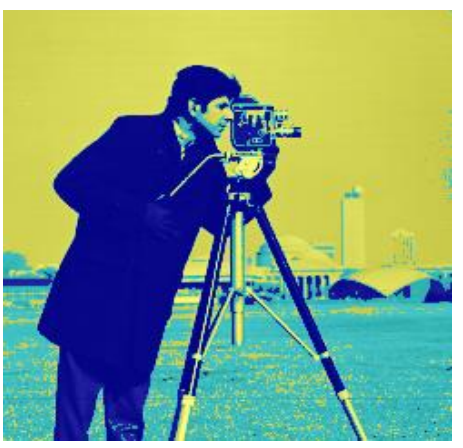

Fig.5 - K-means labelled image

\section{OTSU ALGORITHM VS. K-MEANS METHOD}

On the basis of analysis done it can be said that the Otsu method needs the image to be pre-processed so that the high accuracy can be obtained. Many times Otsu technique diverted to a larger class variance which can cause missing of weak objects in image [8]. Otsu methods shows good performance if the histogram is of bimodal distribution and is inferred to use a sharp valley linking two tops rather than the fact that the object is not so big in comparison of background region, the histogram then not retains the bimodality [3]. In Otsu thresholding if the image is corrupted due to noise then histogram got affected and Otsu method leads to termination due to segmentation error. One of the biggest limitation of the Otsu method is that it considers only two classes in histogram but most of the time there are more than two classes of pixels in a digital image so in that condition the Otsu method does not fits suitable for segmentation.

$\mathrm{K}$-means method of image segmentation is a technique which does not use the histograms for the segmentation process that's why the noise introduced in image can be avoided. In $\mathrm{K}$-means the different pixels are evaluated and similar pixels or data sets are grouped together. Since K-means is suitable for large data sets and it works on spherical clusters, it is efficient, flexible to make change. In comparison to other segmentation methods K-means approach of image segmentation is fast and efficient in terms of computational cost. One of the major drawback of the K-means approach is uniform effect, which refers the outcome of same size clusters while the input data is of different sizes. In K-means it is also difficult to predict the Value of $\mathrm{k}$ which is need to be specified in initial phase of algorithm.

\section{CONCLUSION}

The Otsu algorithm and k-means method is reviewed with its working and limitations. Because of simple nature of Otsu algorithm it is being used widely but it has some limitations too, so many improvements in Otsu algorithm have also made. No single method of image segmentation is enough for all sort of digital images and all methods are not suitable for a particular image type. Otsu method is not compatible for hazardous images. Likewise $\mathrm{K}$-means method is also not suitable for many image types like if the image does not form the spherical clusters then it can work improperly and outcome may be affected. Hence to overcome these drawbacks of Otsu algorithm various enhancements are also made and further work can also be carried out.

\section{REFERENCES}

[1]Rafael C. Gonzalez, Richard E. Woods, “ Digital Image Processing”, $2^{\text {nd }}$ ed., Beijing: Publishing House of Electronics Industry, 2007.

[2]Dilpreet Kaur and Yadwinder Kaur, "Image Segmentation Techniques A Review", International Journal of Computer Science and Mobile Computing, Vol.3 Issue 5, May-2014, pg. 809-814.

[3]Er. Nirpjeet Kaur and Er. Rajpreet Kaur, "A review on various method of image thresholding", IJCSE-2011.

[4]Zhong Qu and Li Hang research on " Image Segmentation Based on the Improved Otsu Algorithm”, 2010.

[5]D. Liu and J. Yu, "Otsu method and K-means", 2009 Ninth International Conference on Hybrid Intelligent Systems, Shenyang, 2009, pp. 344-349.

[6]Himanshu Makkar and Aditya Pundir, "Image Analysis using Improved Otsu Thresholding Method", International Journal on Recent and Innovation Trends in Computing and Communication, pp. 2122-2126.

[7] Nameirakpam Dhanachandra, Khumanthem Manglem, Yambem Jina Chanu, "Image Segmentation Using K -means Clustering Algorithm and Subtractive Clustering Algorithm", Procedia Computer Science, Volume 54, 2015, Pages 764-771, ISSN 1877-0509.

[8]Prof. Sushilkumar N. Holambe, Priyanka G. Kumbhar, "Comparison between Otsu's Image Thresholding Technique and Iterative Triclass", International Journal of Computer Trends and Technology (IJCTT)Volume 33 Number 2- March 2016.

[9] W. K. Pratt, "Image Segmentation", in Digital Image Processing, $4^{\text {th }}$ ed. Wiely, 2008.

[10] Hetal J. Vala and Astha Baxi, “ A Review on Otsu Segmentation Algorithm”, IJARCET, Vol. 2, Issue 2, February 2013.

[11] Otsu N., "A Threshold Selection Method from Grey Level histogram”, IEEE Trans. On System Man Cybernetics, 1979, Vol. 9, No. 1, pp. 62-66.

[12] W. X. Kang, Q. Q. Yang, R. R. Liang, “ The Comparative Research on Image Segmentation Algorithms", IEEE Conference on ETCS pp. 703-707, 2009.

Arpan Kumar, M.Tech Scholar, Department of CSE, Bhabha Institute of Technology, Kanpur, India.

Anamika Tiwari, Assistant Professor, Department of CSE, Bhabha Institute of Technology, Kanpur, India. 